\title{
CHANNELING OF RESPONSES ELICITED BY HYPOTHALAMIC STIMULATION*
}

\author{
Elliot S. Valenstein $\dagger$ \\ Department of Psychology and Neurosciences Laboratory, University of Michigan, Ann Arbor \\ Michigan
}

SCIENTIFIC discovery, like mining, often progresses on a discontinuous course. Progress in one direction continues until a point of diminishing returns is reached. A new strike may produce a scurry of activity in another direction. The development of different techniques may prompt the reopening of a closed shaft. Only after a period of such exploration may a more complete picture of the field emerge. The history of the hypothalamus seems to be following such a course.

Prior to the turn of this century, little attention was given to the hypothalamus. The fact that the obesity observed as part of the Fröhlich syndrome was attributed to pituitary difficulties and not related to hypothalamic involvement clearly reflected the prevailing scientific bias. ANDERSON ${ }^{1}$ traced the history of this period and quoted the following portion of Fröhlich's remarks in 1940 at a symposium on the hypothalamus: "The discussions that I have attended the past two days have established the fact that we were wrong in 1901, that it was not the pituitary body but the hypothalamus, but then I must remind you that all we knew at that time was that the hypothalamus was an anatomical region lying beneath the thalamus. That is all we knew."

Following the classic work by KARPLUS and KREIDL, ${ }^{2}$ RANSON ${ }^{3,4}$ and his colleagues, and Hess, ${ }^{5,6}$ the hypothalamus became increasingly stressed as a significant area for the expression of emotion and motivated behavior. The fact that electrical stimulation in this region elicited a number of autonomic responses constituted a major portion of the evidence. Earlier reports emphasized the elicitation of isolated responses such as pupillary dilation and constriction, but there was a gradual awareness that stimulation was capable of activating integrated bodily changes characteristic of such states as rage, temperature regulation, eating and somnolence. For a brief period it was claimed that stimulation activated only the motor aspects of these states and not the motivational or affective components; ${ }^{7}$ however, later work demonstrated that the behavior elicited by hypothalamic stimulation had many properties in common with behavior motivated by more

\footnotetext{
*Supported by National Institute of Mental Health Grant M-4529 and Research Scientist Award MH-4947.

tThe Author is indebted to Mary Cooper, Sylvia Vaughn, Peter Beal and Dr. Verne C. Cox for their very able aid during various stages of this project.
} 
natural conditions. Animals could be trained to perform learned behaviors either to turn stimulation on ${ }^{8}$ or off ${ }^{9}$ as well as to obtain access to a goal object necessary to perform the elicitcd behavior. ${ }^{10}$ These obscrvations, supplemented by additional stimulation studies, have led to the conclusion that there are discrete hypothalamic regions which play a primary role in directing such specific behavior as feeding, drinking, attack, maternal responses, hoarding and sex as well as other responses. Although they are not mutually exclusive, various investigators have given different emphasis to explanations of elicited behavior based on the activation of biological 'drives' such as hunger, the sensitization of the neural substrate of specific motor patterns, or the selective filtering of impinging stimuli.

There is some indication that we may be entering a period of retreat from what may have been an overemphasis on the specificity of hypothalamic control. Evidence from several sources suggest that this may be the case. For example, although FLYNN ${ }^{11}$ has described the elicitation of attack behavior by hypothalamic stimulation, in conjunction with ELLISON $^{12}$ he has pointed out that cats are capable of integrated attack responses following surgical isolation of the hypothalamus. Also, several reports now exist that indicate that extrahypothalamic stimulation at diverse sites is capable of eliciting responses such as eating and drinking which have been traditionally linked with the hypothalamus. ${ }^{13}$ It is very likely to be the case that just as the prevailing bias at the turn of the century led to a neglect of the hypothalamus, the prevailing bias during the past two decades has led to a relative neglect of the rest of the nervous system by investigators interested in motivated behavior. Furthermore, hypothalamic areas believed to be primarily responsible for one behavior category have been implicated in processes that have broader application. In this vein, GROSSMAN ${ }^{14}$ has questioned the validity of restricting the function of the ventromedial hypothalamic nucleus to a food 'satiety' center and has suggested that the behavioral changes observed following destruction of this nucleus " . . may be due to an interference affective rather than appetitive mechanisms."

More recently, other evidence ${ }^{15,16}$ has been presented which supports the view that it may be necessary to re-examine some of the conclusions based on electrical stimulation studies. Briefly, this evidence has supported the following conclusions: (1) Elicited behavior differs significantly from behavior evoked by motivational states such as hunger and thirst; (2) If sufficient opportunity is provided, the majority of hypothalamic electrodes elicit several different behaviors. These different behaviors do not necessarily share the same motor elements nor do they appear to be linked by any specific motivational state; and (3) Anatomical locus of electrodes does not provide an adequate basis for predicting the elicited behavior.

The present study was designed to elaborate on some factors responsible for the reliable elicitation of a given behavior pattern by hypothalamic stimulation and some determinants of the ease or difficulty in eliciting other responses from the same electrode.

\section{SUBJECTS AND METHODS}

Male and female Sprague-Dawley rats (250-350 g) purchased from the Holtzman Company were implanted with electrodes in the lateral hypothalamus and following 
recovery from surgery, animals were tested to determine if electrical stimulation elicited eating or drinking (stimulus-bound behavior). Stimulation consisted of $60 \mathrm{c} / \mathrm{s}$ sine wave with currents ranging during the initial screening between 3 and $25 \mu$ A (R.M.S.). Animals displaying stimulus-bound eating or drinking were used in one of two experiments. It is important to note that once an intensity was found which elicited either eating or drinking, this same intensity was always used on all future tests. All testing and screening was done when animals were satiated for food and water and displayed no tendency to eat or drink unless stimulated.

\section{EXPERIMENT 1}

The purpose of this experiment was to determine if the amount of opportunity provided to display a particular elicited behavior would influence the difficulty of acquiring a new behavior in response to the same stimulation. Eighteen animals were used in this experiment and they were assigned at random to either the Control Group $(\mathrm{N}=6)$ or the ExtraElicitation Group $(\mathrm{N}=12)$. After it was determined that an animal displayed either eating or drinking in response to stimulation, all animals received two Standard Tests. A Standard Test consisted of twenty 30 -sec stimulation periods separated by a 60 -sec interstimulus interval. In some of these tests (Two Goal Object) both food and water were available, and in others (One Goal Object) only one of these was offered to the animal. The food used was Purina Lab Chow pellets scattered on the floor; water was presented in a single bottle mounted so that the drinking tube extended about two inches into the Plexiglas test chamber. The behavior was scored by an observer, who recorded the occurrence of eating only when it was evident that the rat was biting off and ingesting pieces of the food pellets and drinking when the tube was lapped and water ingested.

All 18 animals were given two Standard Tests with both food and water presented and were selected from a larger group on the basis that only one behavior (either eating or drinking) was elicited by the stimulation. All animals displayed either eating or drinking during at least 75 per cent of the stimulation presentations and did not eat or drink during any of the interstimulus intervals.

The Extra-Elicitation Group was then given 30 Standard Tests with only the goal object to which they oriented present in the chamber. Thus, in these One Goal Object Tests if an animal had previously displayed only eating in response to stimulation, food was the only goal object present. The converse was true for animals that had only displayed elicited drinking. The number of tests per day varied, but never exceeded three and they were always spaced at least $1 \mathrm{hr}$ apart.

Animals in the Control Group were placed in the test chamber a comparable period of time, with the cable connected to their electrode, but no stimulation was presented. Following these tests (or pseudo tests) all animals were tested with the opposite goal object so that animals which had displayed only eating in response to stimulation were offered only the water bottle. Animals received a 30-sec stimulation train every $90 \mathrm{sec}$ and an observer recorded the occurrence of any behavior. After each hour of stimulation the animals were given a $15 \mathrm{~min}$ rest from stimulation with both food and water available. Animals were also given $3-4 \mathrm{hr}$ to rest after $12 \mathrm{hr}$ on the above schedule. This sequence 
continued until the animal had displayed the new behavior (either eating or drinking) in response to stimulation on at least 3 out of 5 consectutive stimulus trains. The number of stimulations necessary to reach this criterion was rccorded. If a new behavior was not in evidence after 1500 stimulations, the testing was discontinued and it was considered that the animal had not exhibited a new stimulus-bound behavior pattern.

Following the emergence of the new elicited behavior, the animals were given a Standard Test with only the goal object relevant to the new behavior present. The purpose of this test was to assure that the new behavior was elicited reliably. In every instance stimulation elicited the new behavior on at least 75 per cent (Av. 90 per cent; Range 75-100 per cent) of the presentations. All animals were then given a competitive Two Goal Object Standard Test to determine the relative strengths of the initial and new elicited behaviors.

Table 1. Difficulty of establishing a NeW stimulus-bound behavior pattern as a fUNCTION OF FREQUENCY OF ELICITATION OF ORIGINAL BEHAVIOR

\begin{tabular}{|c|c|c|c|c|}
\hline & \multirow{2}{*}{$\begin{array}{l}\text { Av. No. stimulations } \\
\text { prior to new } \\
\text { behavior }\end{array}$} & \multirow{2}{*}{$\begin{array}{c}\text { Per cent } \\
\text { animals } \\
\text { exhibiting } \\
\text { newbehavior* }\end{array}$} & \multicolumn{2}{|c|}{ Behavior elicited in competition tes } \\
\hline & & & $\begin{array}{c}\text { Per cent } \\
\text { original behav. }\end{array}$ & $\begin{array}{l}\text { Per cent } \\
\text { new behav. }\end{array}$ \\
\hline $\begin{array}{l}\text { Extra-elicitation } \\
\text { group }(\mathrm{N}=12)\end{array}$ & 500 & 67 & 90 & 10 \\
\hline $\begin{array}{l}\text { Control } \\
\quad \text { group }(N=6)\end{array}$ & 167 & 100 & 42 & 58 \\
\hline
\end{tabular}

* If a new behavior was not elicited after 1500 stimulations, the animal was scored as not exhibiting new behavior.

\section{Results}

The major findings are summarized in Table 1. It can be seen that the animals which received the extra-elicitation of the original behavior were more resistant to the development and display of a new behavior in response to stimulation. It was only necessary to provide an average of 167 stimulations in order to obtain the reliable elicitation of a new behavior in all the control animals. In contrast, only 67 per cent of the animals in the extra-elicitation group exhibited a new behavior within the limits of our testing and in these instances it was necessary to provide an average of 500 stimulations. Furthermore, when the new and initial behaviors were tested in a competitive stimulation with both food and water available, the control animals exhibited the new behavior in the majority of the instances while the new behavior displayed by the animals in the extra-elicitation group constituted only 10 per cent of the total elicited behavior (Table 1 ).

\section{EXPERIMENT 2}

It was observed that in the majority of instances in which a behavior was elicited by stimulation there seemed to be an initial period during which the elicitation of the behavior became increasingly more reliable. It was the purpose of this experiment to study this process more systematically. 
Except where otherwise noted, the experimental methodology was the same as described in the first experiment. Specifically, surgical procedures, stimulation parameters, Standard Tests and subject population were identical to those used in Experiment 1. Two groups of naive animals were compared. The Minimal Stimulation Group $(\mathrm{N}=5)$ consisted of animals which had received less than 40 stimulation trains before receiving a 2 week 'rest period' without stimulation. Prior to the rest period these animals were stimulated only until three instances of either eating or drinking were elicited, but they were not used if this criterion was not met prior to the presentation of 40 stimulations. During this initial screening, the stimulus intensity was gradually raised until, judging by the animal's behavior, the probability of eliciting eating or drinking was maximal. Animals in the Control Group $(\mathrm{N}=8)$ were given a series of standard tests until they had succeeded in exhibiting elicited behavior on at least 50 per cent (10 out of 20 ) of the stimulations on 2 consecutive tests. For 1 animal in this group this criterion was reached in 2 Standard Tests, but most required more and 3 subjects received 11 tests. Following the achievement of this criterion the control animals were also given a 2 week respite from stimulation.

After the 2 week rest period, animals in both groups were given standard tests until they exhibited an elicited behavior during 50 per cent of the stimulation periods on two consecutive tests. At the completion of this sequence the animals received slightly different treatment depending upon their performance. In the case of animals that were exhibiting both eating and drinking, the behavior that was exhibited less frequently was selected for additional testing with only one goal object present. Thirty standard tests were given with tests separated by at least $1 \mathrm{hr}$ and no more than three of these one goal object tests given on a single day. Thus, an animal which exhibited less eating than drinking in the two goal object tests following the 2 week rest period was given 30 tests with only food available. Animals that exhibited only one behavior in the two goal object test were stimulated until a new behavior appeared and then they were also given 30 tests with only the goal object of the weaker behavior present. After the 30 tests the animals were given a series of standard tests with both goal objects present until it was clear which behavior was exhibited more frequently.

\section{Results}

Figure 1 compares the effect of the 2-week rest period without stimulation following different amounts of initial stimulus-bound behavior experience. It can be seen that animals in the Minimal Stimulation Group, which received only a brief initial screening, exhibit very little stimulus-bound behavior immediately after the rest period. With each successive test after the rest period, the animals in this group exhibit a greater amount of elicited behavior. In contrast, animals in the Control Group displayed a relatively high amount of stimulus-bound behavior immediately after the rest period. These animals received a greater amount of opportunity to display stimulus-bound behavior prior to the rest period, and their performance after 2 weeks was almost exactly what it had been on the last test. The Control Group animals also tended to increase the amount of stimulus-bound behavior displayed on the second test after the rest period, but they were not tested further, as all had achieved criterion level. 


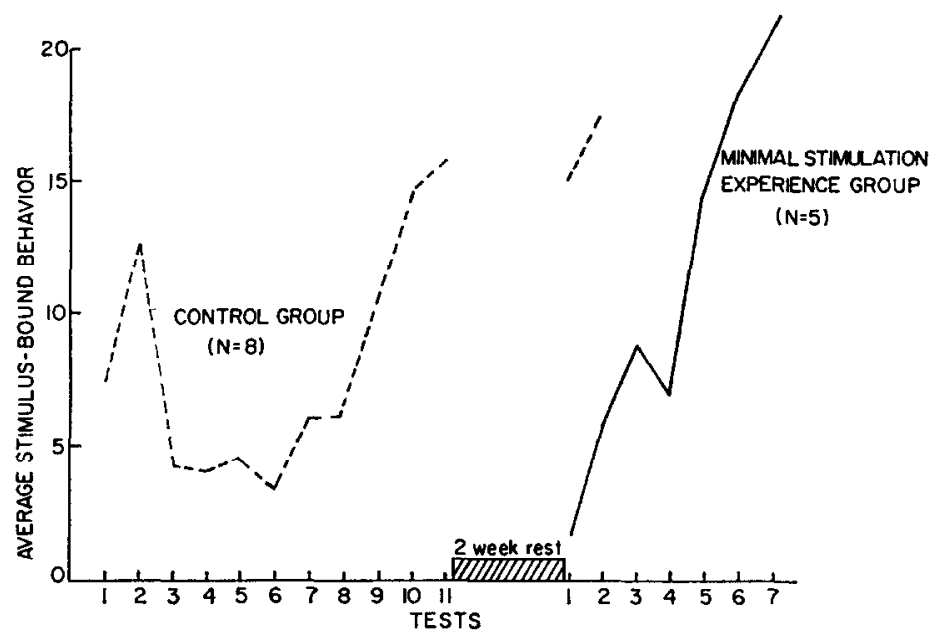

Fig. 1. Comparison of the effect of a 2-week rest period without stimulation, following different amounts of experience. Prior to the rest period, animals in the Minimal Stimulation Group received only a brief initial screening consisting of less than 40 stimulations (eating and/or drinking were elicited only 3 times). Animals in the Control Group received a series of standard tests until they achieved criterion (cf. text). The variability in the curve of the control group prior to the rest period was caused by a reduction in the number of animals as criterion was achieved. All individual records were consistent with the trend depicted by the group averages (cf. Fig. 2). Twenty stimulations were administered on each test. Animals displaying stimulusbound eating and drinking could achieve a score above 20 if both behaviors were displayed during stimulus periods.

Figure 2 illustrates the performance of an individual animal from the Minimal Stimulation Group and an individual animal from the Control Group. The performance of animal $14 \mathrm{JJ}$ is consistent with the group average illustrated in Fig. 1. This animal received only the minimal stimulation involved in the initial screening, and after the 2-week rest period stimulus-bound eating was not evident at all on the first test. Gradually over successive tests, eating was elicited by stimulation more and more reliably. No stimulus-bound drinking was observed even though the water bottle was available. Following these tests, stimulation in the presence of the water bottle, but with no food available, was continued until some evidence of stimulus-bound drinking was observed. Thirty tests with only the water bottle available were then administered, and it can be seen from Fig. 2 that drinking was elicited more and more reliably over this testing sequence. In the competitive tests with food and water both available, which followed the 30 single goal object test, stimulusbound drinking tended to be displayed more frequently. Thus a behavior that was not seen at all during the initial competitive tests became the more dominant pattern.

The tendency for repeated testing of one stimulus-bound behavior pattern to produce a dominance was not always evident, as can be noted from the performance of animal $71 \mathrm{JJ}$ (Fig. 2). This animal was in the Control Group and it can be seen that prior to the 2-week rest period stimulus-bound drinking was elicited more reliably over successive tests. 


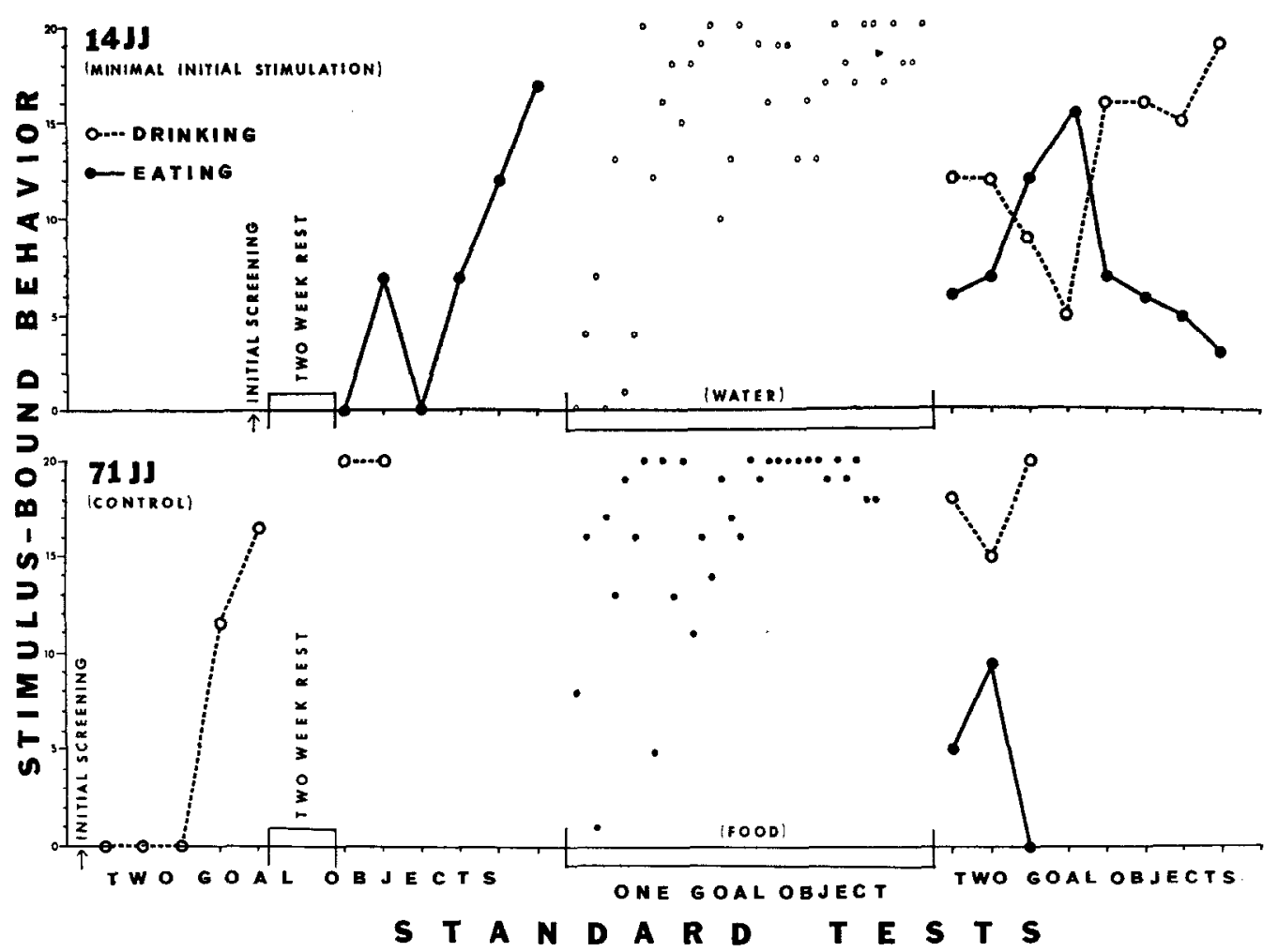

FIG. 2. Individual animal records illustrating effect of a 2-week rest period following different amounts of experience. Also shown are two different results of attempts to strengthen weaker behavior by providing 30 additional standard tests with only one goal object prior to competitive tests with both goal objects (cf. text).

At the end of the rest period a high level of stimulus-bound drinking was exhibited immediately. As described for 14JJ, a new stimulus-bound behavior (eating) gradually emerged and then was observed to be elicited more reliably over the successive 30 single goal object tests. In this instance, however, the new behavior was elicited less frequently in the final competitive tests. No pattern could be described that permitted a prediction of the behavior that dominated in the final competitive tests. In some cases the new and in others the initial pattern dominated and this seemed to be independent of the testing history of the animal.

\section{DISCUSSION}

Several important points emerge from the results of the two experiments described in the present report. It is evident from the data obtained in Experiment 1 that the likelihood of observing a second or new behavior pattern elicited by hypothalamic stimulation is 
influenced by the amount of opportunity an animal has had to display only the initially elicited behavior. Animals that have had a great amount of experience displaying a given stimulus-bound behavior pattern are more resistant to adopting a second pattern, as they seem to persist in ignoring other alternatives. These results have both methodological and theoretical implications. With respect to the former, it would seem that there is a definite possibility that the amount of specificity of function attributed to a given hypothalamic area can easily be exaggerated with some testing procedures. This would be particularly true, for example, when interest is restricted to one behavior and only at a later time is it thought important to test for the possibility that additional behaviors might be elicited from activation of the same site. As can be seen from Table 1, if animals are tested for only one behavior it may take more than 500 stimulations before a second behavior starts to be elicited reliably. This is likely to involve considerably more effort than is usually expended in testing for the presence of other stimulus-bound behaviors.

ROBERTS ${ }^{17}$ has argued that the testing of animals with several goal objects simultaneously available may be misleading, as a weaker response to the same stimulation may not have an opportunity to be expressed. He recommends that animals be tested with each object separately before concluding that the stimulation did not have the capacity to elicit a second behavior initially. This is probably good advice, but it should be clear that in the cases we have described there was no evidence that the stimulation had the capacity to elicit the second behavior from the outset. Even when tested with only one goal object available, it characteristically took a large number of stimulations before the second behavior was first displayed and usually it was elicited only during a small percentage of the stimulations. This trend is particularly evident in Experiment 2, where it was noted that the elicitation of stimulus-bound behavior normally went through a process of 'strengthening' (cf. Figs. 1 and 2). After the animal had sufficient opportunity to display the new behavior in response to stimulation it was likely to be exhibited in the competitive situation, often predominantly, in spite of the fact that it had not been displayed at all in the initial competitive tests. It is unlikely that this strengthening process is simply reflecting the animals' increasing familiarity with the testing cage, goal objects or even the location of the goal objects. Animals were always provided with sufficient opportunity to explore the test chamber and the location of the food and water should have posed no problem as the familiar Purina Lab Chow pellets were scattered all over the floor and the water was in the same type of bottle that was attached to the home cage. Animals were also observed eating and drinking on occasion during habituation and rest periods in the chamber without stimulation. The animals in brief seem to know the relevant spatial information prior to stimulation. To obtain the food and water, no unfamiliar instrumental responses were required that could explain the necessity for several hundred (considerably above 500 in many instances) stimulations to be presented before the new stimulus-bound behavior appeared.

Nor is there any reason to believe that the postulation of a developing incentive motivation' would be helpful. In the present context, the term 'incentive motivation' implies that the capacity of the goal objects (food and water) and the testing situation to elicit eating or drinking has been enhanced. There does not seem to be any evidence to support the view that this is a major factor in the emergence of stimulus-bound behavior. VALENSTEIN and $\operatorname{Cox}^{18}$ have shown, for example, that placing animals on a regimen where they received 
all their food and/or water in a distinctive test chamber and at a particular time during the day did not increase the probability of obtaining stimulus-bound behavior by local brain stimulation.

Another conclusion that may be drawn from Experiment 2 is, that once a stimulus-bound behavior pattern becomes well established, it tends to retain a significant position in an animal's response hierarchy even though not exercised repeatedly. It was seen in Fig. 1 that animals in the Control Group which were tested until the percentage of stimulus-bound behavior had reached criterion level, exhibited the same amount of elicited behavior after a 2-week rest period. The same conclusion can be drawn from the fact that when animals have not had an opportunity to display the initial stimulus-bound behavior for an extended period during the establishment and 'strengthening' of a second behavior, the initial pattern is generally still displayed in later competitive tests (Fig. 2).

It is difficult to prove conclusively that all the stimulus-bound patterns that eventually emerge are not reflecting pre-existing connections between the hypothalamic area stimulated and the neural substrate underlying the elicitation of the responses. Our own experience with this phenomenon has led us to conclude that it is unnecessary to make such an assumption. The many instances we have observed where a stimulus-bound behavior emerges only after a very long period of testing, and then gradually becomes elicited with greater frequency suggests that in some ways an association is being developed. It seems to us that the assumption that there always exists a fixed association between the hypothalamic area stimulated (or the state induced by such stimulation) and the specific expression of a particular response pattern that finally is clicited, is gratuitous. It is not implicd that any arbitrarily selected response can be associated with stimulation at any hypothalamic site, and indeed some limitations have been discussed by the author elsewhere. ${ }^{19}$ It is suggested, however, that evidence is accumulating which indicates that it is possible to establish and strengthen the association between the effects of hypothalamic stimulation and a specific mode of expression. The mode of expression has to be consistent with the state induced by stimulation, but it is not predetermined. This is what is implied by the title: 'Channeling of Responses Elicited by Hypothalamic Stimulation.'

It may not be accidental that most stimulus-bound behaviors that have been studied have been observed in conjunction with the stimulation of hypothalamic sites judged to be 'reinforcing' by self-stimulation performance. Once a behavior pattern is elicited with a degree of consistency, this response seems to be highly resistant to extinction. Perhaps the association of certain response patterns with the activation of a neural system involved in a basic reinforcement process creates the underlying condition for the maintenance of the behavior when the animal is in the same state. This is what may be involved in stereotyped, compulsive behaviors.

\section{REFERENCES}

1. Anderson, E. Earlier ideas of hypothalamic function, including irrelevant concepts. In: The Hypothalamus. Haymaker, W., Anderson, E., Nauta, W. (Eds.) Charles Thomas, Springfield, IIl., 1969.

2. KArplus, J. P. and KreidL, A. Gehirn und Sympathicus. I. Mitteilung: Zwischenhirnbasis und Halssympathicus. Pfiugers Arch. ges. Physiol. 129, 138, 1909. 
3. Ransom, S. W. Some functions of the hypothalamus. The Harvey Lectures, 1936-1937, p. 241 Williams \& Wilkins, Baltimore, 1937.

4. Ranson, S. W. and Magoun, H. W. The hypothalamus. Ergebn. Physiol. 41, 56, 1939.

5. Hess, W. R. Diencephalon. Autonomic and Extrapyramidal Function. Grune \& Stratton, New York, 1954.

6. GLOOR, P. Autonomic functions of the diencephalon. A summary of the experimental work of Prof. W. R. Hess. A.M.A. Archs Neurol. Psychiatry 71, 773, 1954.

7. Masserman, J. H. Is the hypothalamus a center for emotion? Psychosom. Med. 3, 1, 1941.

8. OlDS, J. and MILNER, P. Positive reinforcement produced by electrical stimulation of septal area and other regions of rat brain. J. comp. physiol. Psychol. 47, 419, 1954.

9. Delgado, J. M. R., Roberts, W. W. and Miller, N. E. Learning motivated by electrical stimulation of the brain. Am. J. Physiol. 179, 587, 1954.

10. Coons, E. E., Levak, M. and Miller, N. E. Lateral hypothalamus: Learning of food-seeking response motivated by electrical stimulation. Science 159, 1117, 1968.

11. FlynN, J. P. The neural basis of aggression in cats. In: Neurophysiology and Emotion, GLASs, D. C. (Ed.) Rockefeller Univ. Press, New York, 1957.

12. Ellison, G. D. and FLYNN, J. P. Organized aggressive behavior in cats after surgical isolation of the hypothalamus. Archs ital. Biol. 106, 1, 1968.

13. Robinson, B. W. and Mishkin, M. Alimentary responses to forebrain stimulation in monkey. Expl. Brain Res. 4, 330, 1968.

14. Grossman, S. P. The VMH: A center for affective reactions, satiety, or both? Physiol. Behav. $1,1,1966$.

15. VAlENSTEIN, E. S. Behavior elicited by hypothalamic stimulation: A prepotency hypothesis. Brain Behav. Evol. 2, 295, 1969.

16. Valenstein, E. S., Cox, V. C. and Kakolewski, J. W. Reexamination of the role of the hypothalamus in motivation. Psychol. Rev. 77, 16, 1970.

17. RoBerts, W. W. Are hypothalamic motivational mechanisms functionally and anatomically specific? Brain Behav. Evol. 2, 317, 1969.

18. Valenstein, E. S. and Cox, V. C. Influence of hunger, thirst and previous experience in the test chamber on stimulus-bound eating and drinking. J. comp. physiol. Psychol. 70, 189, 1970.

19. Valenstein, E. S. Behavior elicited by hypothalamic stimulation: A prepotency hypothesis. Brain Behav. Evol. 2, 295, 1969. 